\title{
Systematic Development of Correct Bulk Synchronous Parallel Programs
}

\author{
Louis Gesbert*, Zhenjiang $\mathrm{Hu}^{\dagger}$, Frédéric Loulergue ${ }^{\ddagger}$, Kiminori Matsuzaki ${ }^{\S}$ and Julien Tesson $₫$ \\ *MLstate, Paris, France, louis.gesbert@mlstate.com \\ $\dagger^{\dagger}$ National Institute of Informatics, Tokyo, Japan, hu@nii.ac.jp \\ ‡LIFO, Université d’Orléans, France, Frederic.Loulergue@univ-orleans.fr \\ $\S$ Kochi University of Technology, Tokyo, Japan, matsuzaki.kiminori@kochi-tech.ac.jp \\ `LIFO, Université d'Orléans, France, Julien.Tesson@univ-orleans.fr
}

\begin{abstract}
With the current generalisation of parallel architectures arises the concern of applying formal methods to parallelism. The complexity of parallel, compared to sequential, programs makes them more error-prone and difficult to verify. Bulk Synchronous Parallelism (BSP) is a model of computation which offers a high degree of abstraction like PRAM models but yet a realistic cost model based on a structured parallelism. We propose a framework for refining a sequential specification toward a functional BSP program, the whole process being done with the help of the Coq proof assistant. To do so we define BH, a new homomorphic skeleton, which captures the essence of BSP computation in an algorithmic level, and also serves as a bridge in mapping from high level specification to low level BSP parallel programs.
\end{abstract}

\section{INTRODUCTION}

With the current generalisation of parallel architectures and increasing requirement of parallel computation arises the concern of applying formal methods, which allow specifications of parallel and distributed programs to be precisely stated and the conformance of an implementation to be verified using mathematical techniques. However, the complexity of parallel programs, compared to sequential ones, makes them more error-prone and difficult to verify. This calls for a strongly structured form of parallelism [18], [22], which should not only be equipped with an abstraction or model that conceals much of the complexity of parallel computation, but also provide a systematic way of developing such parallelism from specifications for practically nontrivial examples.

The Bulk Synchronous Parallel (BSP) model is a model for general-purpose, architecture-independent parallel programming [10], [28]. The BSP model consists of three components, namely a set of processors each with a local memory, a communication network, and a mechanism for globally synchronising the processors. A BSP program proceeds as a series of super-steps. In each super-step, a processor may operate only on values stored in local memory. Values sent through the communication network are guaranteed to arrive at the end of a super-step. Although the BSP model is simple and concise, it remains as a challenge to systematically develop efficient and correct BSP programs that meet given specifications.

To see this clear, consider the following tower-building problem, which is an extension of the known line-of-sight problem [4]. Given a list of locations (position $x_{i}$ and height



Fig. 1

TOWER-BUILDING PROBLEM

$h_{i}$ ) along a line in the mountain (see Fig. 1):

$$
\left[\left(x_{1}, h_{1}\right), \ldots,\left(x_{i}, h_{i}\right), \ldots,\left(x_{n}, h_{n}\right)\right]
$$

and two special points $\left(x_{L}, h_{L}\right)$ and $\left(x_{R}, h_{R}\right)$ on the left and right of these locations along the same line, the problem is to find all locations from which one can see the two points after building a tower of height $h$. If we do not think about efficiency and parallelism, this problem can be easily solved by considering for each location $\left(x_{i}, h_{i}\right)$, whether it can be seen from both $\left(x_{L}, h_{L}\right)$ and $\left(x_{R}, h_{R}\right)$. The tower with height $h$ at location $\left(x_{i}, h_{i}\right)$ can be seen from $\left(x_{L}, h_{L}\right)$ if for any $k=1,2, \ldots, i-1$ the inequality

$$
\frac{h_{k}-h_{L}}{x_{k}-x_{L}}<\frac{h+h_{i}-h_{L}}{x_{i}-x_{L}}
$$

holds. Similarly, it can be seen from $\left(x_{R}, h_{R}\right)$ means that for any $k=i+1, \ldots, n$, the inequality

$$
\frac{h_{k}-h_{R}}{x_{R}-x_{k}}<\frac{h+h_{i}-h_{R}}{x_{R}-x_{i}}
$$

holds. While the specification is clear, its BSP parallel program, say in BSML [8], a library for BSP programming in the functional language Objective Caml, is rather complicated. This gap makes it difficult to verify that the implementation is correct with respect to the specification.

In this paper, we propose the first general framework (in Sect. (II), as far as we are aware, for systematic development of certified functional BSP parallel programs. More specifically, (1) we introduce a novel algorithmic skeleton (Sect. III), BSP Homomorphism (or $\mathrm{BH}$ for short), which can not only capture the essence of BSP computations at algorithmic level, but also serve as a bridge by mapping high level specification to low level BSP parallel programs; (2) we develop a set of useful 


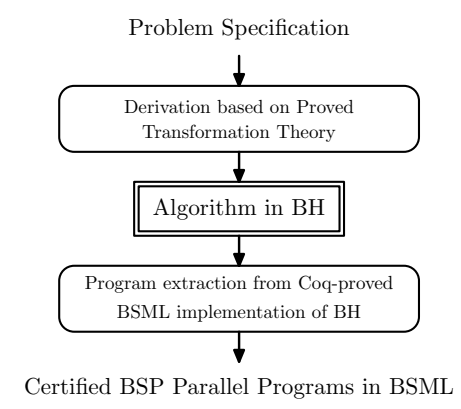

Fig. 2

AN OVERVIEw OF OUR FramewORK FOR DEVEloping CORRECT BSP PROGRAMS

theories (Sect. IV) in Coq for systematic and formal derivation of programs from specification to $B H$, and we provide a certified parallel implementation (Sect. VD of $B H$ in BSML so that a certified BSP parallel program can be automatically extracted; and (3) we demonstrate with examples that our new framework can be very useful to develop certified BSP parallel programs for solving various nontrivial problems (Sect. VI).

\section{AN OVERVIEW}

Figure 2 depicts an overview of our framework. We insert a new layer, called "algorithm in $B H$ ", between problem specifications and certified BSP parallel programs, so as to divide the development process into two easily tacking steps: A formal derivation of algorithms from specification to $B H$ and a proof of correctness of a BSML implementation of $B H$.

In our framework, a specification is described in Coq [26], allowing the user to be confident in its correctness without concern of parallelism. We chose to take Coq definitions as specifications for reasons of simplicity of our system, and for giving access to the full strength of the Coq assistant to prove initial properties of the algorithms (the system will then provide a proof that these properties are preserved throughout the transformations). In the first step, we rewrite the specification into a program using the $B H$ skeleton, in a semi-automated way. To do so, we provide a set of Coq theories over $B H$ and tools to make this transformation easier. This transformation is implemented in Coq, and proved to be correct, i.e. preserving the semantics of the initial specification. Thus, this step converts the original specification into a program (using $\mathrm{BH}$ ) that is proved equivalent. In the second step, we replace the calls to the skeleton $B H$ in the algorithm with a parallel implementation (in BSML) that is proved correct. By using the program extraction features of Coq on the rewritten algorithm, we get a parallel program that implements the algorithm of the specification, and that is proved correct.

\section{A BSP HOMOMORPHISM}

Homomorphisms play an important role in both formal derivation of parallel programs [7], [12], [14], [16] and automatic parallelisation [20]. Function $h$ is said to be a

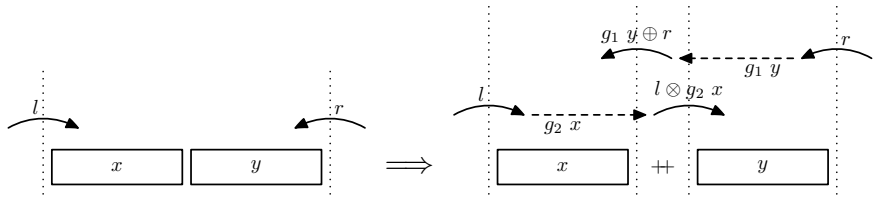

Fig. 3

INFORMATION PROPAGATION FOR $B H$.

homomorphism, if it is defined recursively in the form of

$$
\begin{cases}h[] & =i d \odot \\ h[a] & =f a \\ h(x+y) & =(h x) \odot(h y)\end{cases}
$$

where applying a function $f$ to an expression $x$ is written $f x$, $\left[x_{1}, \ldots, x_{n}\right]$ denotes the list containing the elements $x_{1}$ to $x_{n}$, \# denotes list concatenation, $i d_{\odot}$ denotes the identity unit of $\odot$. Since $h$ is uniquely determined by $f$ and $\odot$, we will write $h=([\odot, f])$.

Though being general, different parallel computation models would require different specific homomorphisms together with a set of specific derivation theories. For instance, the distributed homomorphism [11] is introduced to treat the hyper-cube style of parallel computation, and the accumulative homomorphism [15] is introduced to treat the skeleton parallel computation.

Our BH (BSP Homomorphism) is a specific homomorphism carefully designed for systematic development of certified BSP algorithms. The key point is that we formalise "data waiting" and "synchronisation" in the super-step of the BSP model by computation of gathering necessary information around for each element of a list and then perform computation independently to update each element.

Definition 1 (BSP Homomorphism): Given function $k$, two homomorphisms $g_{1}$ and $g_{2}$, and two associative operators $\oplus$ and $\otimes$, a function $b h$ is said to be a BSP Homomorphism or $B H$, if it is defined in the following way.

$$
\left\{\begin{aligned}
b h[a] l r & =[k a l r] \\
b h(x+y) l r= & b h x l\left(g_{1} y \oplus r\right)++ \\
& b h y\left(l \otimes g_{2} x\right) r
\end{aligned}\right.
$$

The above $b h$ defined with functions $k, g_{1}, g_{2}$, and associative operators $\oplus$ and $\otimes$ is denoted as

$$
b h=B H\left(k,\left(g_{1}, \oplus\right),\left(g_{2}, \otimes\right)\right) .
$$

Function $b h$ is a higher-order homomorphism, which takes a list as input and returns a new list of the same length. In addition to the input list, $b h$ has two additional parameters, $l$ and $r$, which contain necessary information to perform computation on the list. The information of $l$ and $r$, as defined in the second equation and shown in Fig. 3, is propagated from left and right with functions $\left(g_{2}, \otimes\right)$ and $\left(g_{1}, \oplus\right)$ respectively.

It is worth remarking that $\mathrm{BH}$ is powerful; it cannot only describe super-steps of BSP computation, but is also powerful enough to describe various computation including all homo- 
morphisms (map and reduce) (Sect. IV], scans, as well as the BSP algorithms in [10].

\section{Deriving Algorithms in BH}

In this section, we show how to derive correct algorithms in terms of $\mathrm{BH}$ from problem specifications. The specification gives a direct solution to the problem, where one does not need to think about low level parallel computation issues, such as layout of processors, task distribution, data communication. This specification will be transformed into an equivalent algorithm in terms of $\mathrm{BH}$ based on a set of transformation theorems.

\section{A. Specification}

Coq functions are used to write specification, from which an algorithm in $\mathrm{BH}$ is to be derived. Recursions and the well-known collective operators (such as map, fold, and scan) can be used in writing specification. To ease description of computation using data around, we introduce a new collective operator mapAround.

The mapAround, compared to map, describes more interesting independent computation on each element of lists. Intuitively, mapAround is to map a function to each element (of a list) but is allowed to use information of the sublists in the left and right of the element, e.g.,

$$
\begin{aligned}
& \text { mapAround } f\left[x_{1}, x_{2}, \ldots, x_{n}\right]= \\
& {\left[f\left([], x_{1},\left[x_{2}, \ldots, x_{n}\right]\right), f\left(\left[x_{1}\right], x_{2},\left[x_{3}, \ldots, x_{n}\right]\right),\right.} \\
& \left.\quad \ldots, f\left(\left[x_{1}, x_{2}, \ldots, x_{n-1}\right], x_{n},[]\right)\right] .
\end{aligned}
$$

In addition, we provide a set of communication functions such as permute, shiftL, shiftR to redistribute list elements. They are designed to be not only useful for reconstructing lists in the specification level but also equipped with low lever certified BSP implementations.

Example 1 (Specification of the Tower-Building Problem): Recall the tower-building problem in the introduction. We can solve it directly using mapAround, by computing independently on each location and using informations around to decide whether a tower should be built at this location. So our specification can be defined straightforwardly as follows.

$$
\begin{aligned}
& \text { tower }\left(x_{L}, h_{L}\right)\left(x_{R}, h_{R}\right) x s=\text { mapAround visibleLR } x s \\
& \text { where visibleLR }\left(\text { ls, }\left(x_{i}, h_{i}\right), \text { rs }\right)= \\
& \text { visibleL ls } x_{i} \wedge \text { visibleR rs } x_{i} \\
& \text { visibleL ls } x_{i}=\text { maxAngleL ls }<\frac{h+h_{i}-h_{L}}{x-x_{L}} \\
& \text { visibleR } r s x_{i}=\text { maxAngleR } r s<\frac{h+h_{i}-h_{R}}{x_{R}-x}
\end{aligned}
$$

The inner function maxAngleL is to decide whether the left tower can be seen, and is defined as follows (where $a \uparrow b$ returns the bigger of $a$ and $b$ ).

$$
\begin{array}{ll}
\text { maxAngleL }[] & =-\infty \\
\operatorname{maxAngleL}([(x, h)]+x s) & =\frac{h-h_{L}}{x-x_{L}} \uparrow \operatorname{maxAngleL} x \mathrm{~s}
\end{array}
$$

and the function maxAngleR can be similarly defined.

Example 2 (Maximum Prefix Sum Problem Specification):

Consider the maximum prefix sum problem [4], which is to compute the maximum sum of all the prefix sublists. For instance, supposing mps is the function that solves the problem, we have

$$
\operatorname{mps}[1,-1,2]=1 \uparrow(1+(-1)) \uparrow(1+(-1)+2)=2
$$

We have two ways to solve this problem. One is to decompose this problem to smaller ones, each of which can be described with our list functions, and then compose them together. Following this idea, we could solve the maximum prefix sum problem by first enumerating all the prefix sums and then compute their maximum.

$$
\begin{aligned}
& \text { mps }=\text { maximum } \circ \text { psums } \\
& \text { where } \text { maximum }=(\llbracket \uparrow, i d]) \\
& \text { psums }=\operatorname{scan}(+)
\end{aligned}
$$

Certainly, there could be other specification for the same problem. For example, we could solve this problem as by the following recursive function (both leftwards and rightwards).

$$
\begin{cases}\operatorname{mps}[] & =0 \\ \operatorname{mps}([a]+x) & =0 \uparrow(a+\operatorname{mps}(y)) \\ \operatorname{mps}(x++[a]) & =\operatorname{mps}(x) \uparrow(\operatorname{sum}(x)+b)\end{cases}
$$

Here sum can be defined similarly and its definition is omitted here.

\section{B. Theorems for Deriving $\mathrm{BH}$}

Since our specification is a simple combination of collective functions and recursive functions, derivation of a certified BSP parallel program can be reduced to derivation of certified BSP parallel programs for all these functions, because the simple combination is easy to be implemented by composing supersteps in BSP.

While simple communication functions may be mapped directly to certified BSP parallel programs, it is more difficult for the collective functions and recursive functions, which may be parametrised with other functions and have more flexible computation structure. Our idea is to map these functions into $B H$, and then show how $B H$ can be mapped to a certified BSP parallel programs.

First, let us see how to deal with collective functions. The central theorem for this purpose is the following theorem.

Theorem 1 (Parallelisation mapAround with $\mathrm{BH}$ ): For a function

$$
h=\text { mapAround } f
$$

if we can decompose $f$ as $f(l s, x, r s)=k\left(g_{1} l s, x, g_{2} r s\right)$, where $k$ is any function and $g_{i}$ is a composition of a function $p_{i}$ with a homomorphism $h_{i}=\left(\left[\oplus_{i}, k_{i}\right]\right)$, then

$$
\begin{gathered}
h x s=B H\left(k^{\prime},\left(h_{2}, \oplus_{2}\right),\left(h_{1}, \oplus_{1}\right)\right) x s \iota_{\oplus_{1}} \iota_{\oplus_{2}} \\
\text { where }\left\{\begin{array}{l}
k^{\prime}(l, x, r)=k\left(p_{1} l, x, p_{2} r\right) \text { holds, } \\
\iota_{\oplus_{1}} \text { is the (left) unit of } \oplus_{1}, \\
\iota_{\oplus_{2}} \text { is the (right) unit of } \oplus_{2} .
\end{array}\right.
\end{gathered}
$$

Proof: This has been proved by induction on the input list of $h$ with Coq (available in [27]). 
Theorem 1 is general and powerful in the sense that it can parallelise not only mapAround but also other collective functions to $B H$.

For instance, the useful scan computation

$\operatorname{scan}(\oplus)\left[x_{1}, x_{2}, \ldots, x_{n}\right]=\left[x_{1}, x_{1} \oplus x_{2}, \ldots, x_{1} \oplus x_{2} \oplus \cdots \oplus x_{n}\right]$

is a special mapAround: $\operatorname{scan}(\oplus)=$ mapAround $f$ where $f(l s, x, r s)=$ first $([\oplus, i d]) l s, x,[])$ and first returns the first component of a triple.

What is more important is that any homomorphism can be parallelised with $B H$, which allows us to utilise all the theories [7], [12], [14], [16], [20] that have been developed for derivation of homomorphism.

Corollary 1 (Parallelisation Homomorphism with $\mathrm{BH})$ : Any homomorphism $([\oplus, k])$ can be implemented with a $B H$. Proof: Notice that $([\oplus, k])=$ last $\circ$ mapAround $f$ where $f(l s, x, r s)=(([\oplus, k]) x s) \oplus(k x)$. It follows from Theorem 1 that the homomorphism can be parallelised by a $B H$.

Now we consider how to deal with recursive functions. This can be done in two steps. We first use the existing theorems [12], [14], [20] to obtain homomorphisms from recursive definitions, and then use Corollary 1 to get $B H$ for the derived homomorphisms.

It is worth noting that homomorphisms are very important in all our derivations of $B H$, not only because $B H$ itself is a specific homomorphism, but also because many of our derivations go along with derivations of homomorphisms. For example, in Theorem 11 our main theorem, we have to derive homomorphisms so that function $f$ can be defined in a way that the theorem can be applied.

Example 3 (Derivation for the Tower-Building Problem): From the specification given before, we can see that Theorem 1 is applicable with

$$
\begin{aligned}
& \text { visibleLR }(l s, x, r s)=k\left(g_{1} l s, x, g_{2} r s\right) \\
& \text { where } g_{1}=\max A \text { ngleL } \\
& g_{2}=\max \text { AngleR } \\
& k\left(\operatorname{maxl},\left(x_{i}, h_{i}\right), \max r\right)= \\
& \left(\operatorname{maxl}<\frac{h+h_{i}-h_{L}}{x-x_{L}}\right) \wedge\left(\max r<\frac{h+h_{i}-h_{R}}{x_{R}-x}\right)
\end{aligned}
$$

provided that $g_{1}$ and $g_{2}$ can be defined in terms of homomorphisms.

By applying the theorems in [12], [14], [20], we can easily obtain the following two homomorphisms (the detailed derivation is beyond the scope of this paper).

$\max A$ ngle $L=\left(\left[\uparrow, k_{1}\right]\right)$ where $\uparrow=\max ; k_{1}(x, h)=\frac{h-h_{L}}{x-x_{L}}$ maxAngle $R=\left(\left[\uparrow, k_{2}\right]\right)$ where $k_{2}(x, h)=\frac{h_{R}-h}{x_{R}-x}$

Therefore, applying Theorem 1 yields the following result in $\mathrm{BH}$.

tower $\left(x_{L}, h_{L}\right)\left(x_{R}, h_{R}\right) x s=$ $B H(k,(\max A n g l e L, \uparrow),(\max A n g l e R, \uparrow)) x s(-\infty)(-\infty)$ where $k\left(\operatorname{maxl},\left(x_{i}, h_{i}\right), \operatorname{maxr}\right)$ $=\left(\operatorname{maxl}<\frac{h+h_{i}-h_{L}}{x-x_{L}}\right) \wedge\left(\operatorname{maxr}<\frac{h+h_{i}-h_{R}}{x_{R}-x}\right)$
Example 4 (Maximum Prefix Sum Problem Derivation): Derivation of $B H$ from the specification

$$
\begin{aligned}
& \text { mps }=\text { maximum } \circ \text { psums } \\
& \text { where } \text { maximum }=(\llbracket \uparrow, i d]) \\
& \text { psums }=\operatorname{scan}(+)
\end{aligned}
$$

given above is straightforward, because it is a composition of a homomorphism and a scan, both of which can be mapped to $B H$ according to Theorem 1 and Corollary 1

\section{Theorem Implementation in Coq}

The Coq proof assistant [2], [3], [26] is based on the calculus on inductive construction. This calculus is a higherorder typed $\lambda$-calculus. Theorems are types and their proofs are terms of the calculus. The Coq systems helps the user to build the proof terms and offers a language of tactics to do so. Coq is also a functional programming language. In appendix we give a very short introduction to Coq.

We use the Coq proof assistant to prove correct the derivations from a functional specification to a $\mathrm{BH}$ skeleton instantiation. Derivations and parallel term retrieving is automated using a newly introduced feature in Coq: Type classes [24].

The full code can be downloaded [27].

\section{BH to BSML: CeRTIFIEd PARALlelism}

We have until now supposed a certified parallel implementation of $\mathrm{BH}$ on which the algorithms rely. This implementation is realized using Bulk Synchronous Parallel ML (BSML) [8], an efficient BSP [10], [28] parallel language based on Objective Caml and with formal bindings and definitions in Coq. In Coq, we prove the equivalence of the natural specification of $\mathrm{BH}$ with its implementation in BSML, therefore being able to translate the previous $\mathrm{BH}$ certified versions of the algorithms to a parallel, BSML version. We also analyse the parallel performances of this implementation.

\section{A. Bulk Synchronous Parallel ML: An Overview}

a) Bulk synchronous parallelism: A BSP machine can be though as a homogeneous distributed memory machine with a unit able to synchronise all the processors. A BSP program is executed as a sequence of super-steps, each one divided into (at most) three successive and logically disjointed phases: (a) Each processor uses its local data (only) to perform sequential computations and to request data transfers to/from other nodes; (b) the network delivers the requested data transfers; (c) a global synchronisation barrier occurs, making the transferred data available for the next super-step.

The performance of a BSP machine is characterised by 3 parameters: $p$ is the number of processor-memory pairs, $L$ is the time required for a global synchronisation and $g$ is the time for collectively delivering a 1-relation (communication phase where every processor receives/sends at most one word). The network can deliver an $h$-relation in time $g \times h$ for any arity $h$. The BSP parameters can be determined in practice using benchmarks: first a fourth parameters $r$, the computing power of the processors is determined (in flop/s), then some $g^{\prime}$ and $L^{\prime}$ 
are measured in $s /$ word and $s$, and the final BSP parameters are $g=g^{\prime} \times r$ (in flop/word) and $L=L^{\prime} \times r$ (in flop).

The execution time (or cost) of a super-step is thus the sum of the maximal local processing time, of the data delivery time and of the global synchronisation time:

$$
\max _{0 \leq i<\text { bsp_p }} w_{i}+\max _{0 \leq i<\text { bsp_p }} \max \left(h_{i}^{+}, h_{i}^{-}\right) \times g+L
$$

where, at processor $i, w_{i}$ is the local sequential work performed during the computation phase, $h_{i}^{+}$is the size of data sent from $i$ to other processors, and $h_{i}^{-}$the size of the received data by processor $i$ from other processors.

b) BSML parallel vectors: BSML designed as a full language, is currently implemented as a library for the Objective Caml language [8]. BSML offers an access to the parameters of the underlying BSP architecture with the constants: bsp_p (an integer), bsp_g, bsp_l, and bsp_r (floats).

A BSML program is not written in the Single Program Multiple Data (SPMD) style as many parallel programs are, but offers a global view of the parallel program. It is a usual OCaml program plus operations on a parallel data structure. This structure is called parallel vector and it has an abstract polymorphic type 'a par. A parallel vector has a fixed width bsp_p equals to the constant number of processes in a parallel execution. We will informally write $\left\langle x_{0}, \ldots, x_{n-1}\right\rangle$ for a parallel vector of size $n$, which contains the value $x_{i}$ at processor $i$. The nesting of parallel vectors is forbidden. BSML provides four primitives for the manipulation of parallel vectors. For each of these primitives, we will give its signature, its informal semantics, examples of use and its formalisation in Coq.

In the Coq developments of our framework, all the modules related to parallelism are functors that take as argument a module which provides a realization of the semantics of BSML. This semantics is modelled in a module type called BSML_SPECIFICATION. A module type or module signature in Coq is a set of definitions, parameters and axioms, the latter being types without an associated proof terms.

The module type BSML_SPECIFICATION contains : the definition processor of processor names, and associated axioms; the type par of parallel vectors; the axioms which define the semantics of the four parallel primitives of BSML.

A natural processor_max is assumed to be defined. The total number of processor, the BSP parameter bsp_p is the successor of this natural. The type processor is defined as:

Definition processor $:=\{$ pid: nat $\mid$ pid $<$ bsp_p $\}$.

A term of type $\{x: A \mid P x\}$ is a value of type $A$ and a proof that this value verify the property $P$. A value of type processor thus a pair: A natural and a proof that this natural is lower than bsp_p.

The type of parallel vectors is an opaque type

\section{Parameter par: Set $\rightarrow$ Set.}

This means that par could take as argument a type and returns a new type: It is a polymorphic type thus it has as argument the type of the values contained in the vectors. For example, the type for parallel vectors of string could be written par string (and is written string par in BSML).

In the informal semantics above, a parallel vector consists of $p$ values. As the type par is opaque in the formalisation in Coq, to model this we assume that the type par comes with a function get that can access any component of the parallel vector:

Parameter get : $\forall$ A: Set, par $A \rightarrow$ processor $\rightarrow$ A.

Given a type $T$, a parallel vector vec of type par $T$ and a processor $\mathrm{i}$, (get $\mathrm{T}$ vec $\mathrm{i}$ ) is the value held by processor $\mathrm{i}$ in parallel vector vec.

c) Parallel vector creation: The primitive mkpar is used to create parallel vectors. It takes a function $f$ as argument and creates a parallel vector that contains the value of $(f i)$ at processor $i, 0 \leq i<$ bsp_p. The signature of this primitive is: mkpar: (int $\rightarrow$ 'a) $\rightarrow$ 'a par, and informally its semantics could be written as:

$$
\text { mkpar } f=\langle f 0, \ldots, f(\text { bsp_p }-1)\rangle
$$

In the interactive loop of BSML $[8]$ on a machine with 6 processors, one could have the following result:

\# let this = mkpar (fun $\mathrm{i} \rightarrow \mathrm{i}$ ); ;

val this : int Bsml.par $=\langle 0,1,2,3,4,5\rangle$

\# is the prompt inviting us to write an expression to evaluate. The expression let this $=\ldots$ is used to define the value this by applying the primitive mkpar to the (anonymous) identity function. In the second line, the top-level answers that a value (val) called this has been defined, that it is a parallel vector of integers (int Bsml.par) and its value is $\langle 0,1,2,3,4,5\rangle$.

A useful function is replicate:

\# let replicate $=$ fun $\mathrm{x} \rightarrow \operatorname{mkpar}\left(\right.$ fun $\left._{-} \rightarrow \mathrm{x}\right)$;

val replicate : 'a $\rightarrow$ 'a Bsml.par $=\langle$ fun $>$

\# let $v x=$ replicate "PDCAT";;

vx : string Bsml.par = <"PDCAT", "PDCAT", "PDCAT", "PDCAT", "PDCAT", "PDCAT">

The evaluation of an application of mkpar to a function $f$ is done without any communication (any usual Objective Caml value is replicated on all the processors, hence the function $\mathrm{f}$ ), and is done during the asynchronous computation phase of a BSP super-step. Its BSP cost is $\max _{0 \leq i<\text { bsp_p }} \bar{f} i$ where $\bar{e}$ denotes the time required to evaluate expression $e$.

The semantics of the parallel primitives of BSML in Coq are specified using the get function. It is a quite straightforward translation of the informal semantics. Instead of giving the result parallel vector as a whole it is described by giving the values of its components. A Coq formalisation of the mkpar primitive is thus:

Parameter mkpar_specification : $\forall(A$ :Set) (f: processor $\rightarrow A$ ), $\{X: \operatorname{par} A \mid \forall i$ : processor, get $X i=f i\}$.

\footnotetext{
${ }^{1}$ In case the reader wants to try the examples in the interactive loop (command bsml) while reading the paper, she needs to write open Bsml;; at the beginning of the session.
} 
d) Point-wise parallel application: As the type par and the primitive mkpar are polymorphic, it is possible to create parallel vectors of functions. For example:

\# let vf = mkpar(fun $\mathrm{i} \rightarrow$ fun $\mathrm{x} \rightarrow \mathrm{x}^{\wedge}$ (string_of_int $\left.\mathrm{i}\right)$ ); ;

val vf : (string $\rightarrow$ string) Bsml.par $=\ll$ fun $>, \ldots,<$ fun $\gg$

Thus we may need to apply a parallel vector of functions to a parallel vector of values. Such an application is not a usual functional application: We need a primitive to perform it. This primitive is called apply. Its signature is: apply:('a $\rightarrow$ 'b)par $\rightarrow$ 'a par $\rightarrow$ 'b par.

For example, we can apply the parallel vector of functions vf to the parallel vector of values vx:

\# let v2 = apply vf vx;

val v2 : string Bsml.par = <"PDCAT0", "PDCAT1", "PDCAT2", "PDCAT3", "PDCAT4", "PDCAT5">

The informal semantics of apply could be written as:

apply $\left\langle f_{0}, \ldots, f_{p-1}\right\rangle\left\langle x_{0}, \ldots, x_{p-1}\right\rangle=\left\langle f_{0} x_{0}, \ldots, f_{p-1} x_{p-1}\right\rangle$

The evaluation of an application of apply requires no communication. Its BSP cost is $\max _{0 \leq i<\text { bsp_p }} \overline{f_{i} x_{i}}$. In Coq, apply is specified as follows:

Parameter apply_specification :

$\forall($ A B: Set) (vf: par $(A \rightarrow B))(v x:$ par A),

$\{X: \operatorname{par} B \mid \forall i$ : processor, get $X i=($ get $v f i)$ (get $v x i)\}$.

Very useful functions which are part of the BSML standard library could be implemented from mkpar and apply such as:

(* parfun: ('a $\rightarrow$ 'b) $\rightarrow$ 'a par $\rightarrow$ 'b par $*$ )

let parfun $=$ fun $f \mathrm{v} \rightarrow$ apply (replicate $\mathrm{f}$ ) $\mathrm{v}$

There exist variants parfunN of parfun for point-wisely applying a sequential function with $\mathrm{N}$ arguments to $\mathrm{N}$ parallel vectors.

e) Communications: The two last primitives of BSML require both communication and synchronisation.

The primitive put is used to exchange data between processes. It takes and returns a parallel vector of functions. At each processor the input function describes the messages to be sent to other processors and the output function describes the messages received from other processors. Its signature is put:(int $\rightarrow$ 'a)par $\rightarrow$ (int $\rightarrow$ 'a)par and it informal semantics follows:

$$
\text { put }\left\langle f_{0}, \ldots, f_{p-1}\right\rangle=\left\langle\lambda i . f_{i} 0, \ldots, \lambda i . f_{i}(p-1)\right\rangle
$$

At processor $i$, the function $f_{i}$ encodes the bsp_p possible messages to be sent to other processors. Some values, as the empty list or the first constructor without parameters in a sum type, are considered to mean "no message". For example if we want to broadcast a value at a specific processor in a parallel vector to all other processors, we will use two functions: One that will send this value to every processor, one that will send nothing to every processor. The first of these functions should be used by the processor that is the root of the broadcast, and the second function by all other processors: let choice root pid value $=$

let toall $=$ fun dst $\rightarrow$ [value]

and nothing $=$ fun dst $\rightarrow$ [] in

if pid=root then toall else nothing

Then after the function choice is used to build a parallel vector of functions describing the communications we want to perform, and this parallel vector is given as argument to put, we need to retrieve the sent values. Here again the bsp_p possible received values by a processor are encoded as a function. To know the value sent by a processor $i$ to a processor $j$, one has to apply, at processor $j$, the obtained function to $i$. In the case of the broadcast, all processors need to apply the function to the processor root:



\# let $v 3$ = broadcast 3 v2;;

val v3 : string Bsml.par = <"PDCAT3", "PDCAT3", "РDCAT3", "PDCAT3", "PDCAT3", "PDCAT3">

The evaluation of an application of the put primitive requires a full super-step: First the messages are computed from the parallel vector of functions describing the messages to send; then the messages are exchanged; and a global synchronisation ends the super-step in order to allow the functions describing the received messages to be built. In the following, we note $|v|$ the size (in words) of the value $v$, and $(e) \downarrow$ the result of the evaluation of the expression $e$. The BSP cost of put is:

$$
\begin{gathered}
\max _{0 \leq i<\text { bsp_p }}\left(\sum_{j=0}^{\text {bsp_p-1 } \left.\overline{f_{i} j}\right)}+\max _{0 \leq i<\text { bsp_p }}\left(h_{i}^{+}, h_{i}^{-}\right) \times g+L\right. \\
\text { where }\left\{\begin{array}{l}
h_{i}^{+}=\sum_{0 \leq j<\text { bsp_p }}^{j \neq i}\left|\left(f_{i} j\right) \downarrow\right| \\
h_{i}^{-}=\sum_{0 \leq j<\text { bsp_p }}^{j \neq i}\left|\left(f_{j} i\right) \downarrow\right|
\end{array}\right.
\end{gathered}
$$

$h_{i}^{+}$is the total size of the messages sent by processor $i$, while $h_{i}^{-}$is the total size of the messages received by processor $i$.

The Coq specification of put is:

Parameter put_specification :

$\forall(A$ :Set $)$ (vf: par (processor $\rightarrow A)$ ),

$\{\mathrm{X}: \operatorname{par}$ (processor $\rightarrow A) \mid \forall \mathrm{i}$ : processor, get $\mathrm{X} \mathrm{i} j=$ get $v f \mathrm{j} i\}$.

The last primitive proj:'a par $\rightarrow$ (int $\rightarrow$ 'a) is the inverse of mkpar (for functions defined on the domain of processor names). It also requires a full super-step, we omit the details for the sake of conciseness.

\section{B. $B H$ in $B S M L$}

As a larger BSML example, we present in Fig. 4 a parallel implementation of the $\mathrm{BH}$ algorithmic skeleton where sequence $n 1 n 2$ returns the list $[n 1 ; \ldots ; n 2]$, bh_seq is a sequential implementation of $\mathrm{BH}$ and type of communicated data is type ('l,'r) comm_type = Lcx of 'l | Rcx of 'r | Ncx.

Given a list that is split into local chunks in the order of the processors, the preliminary local computation (applying $\mathrm{gl}$ and gr) can be done with the apply primitive; then a put is used to 


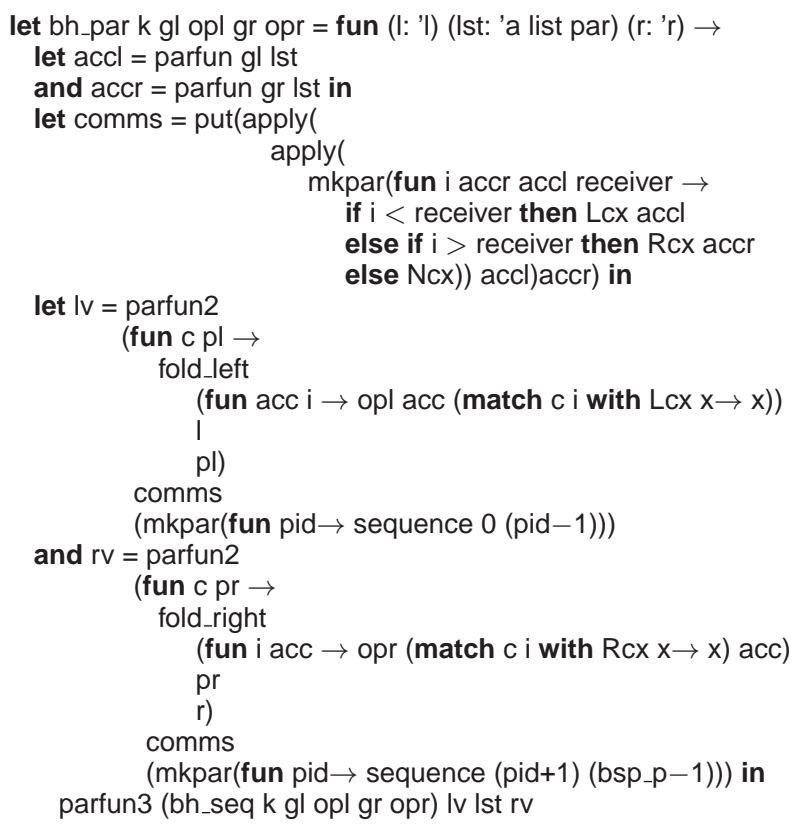

Fig. 4

AN IMPLEMENTATION OF BH IN BSML

send the computed $r$ value leftwards to every other processor, and the I value rightwards. apply is then sufficient to locally compute $\mathrm{BH}$ on the local chunks using the sequential version of $\mathrm{BH}$.

However this implementation is not proved correct to the definition of $\mathrm{BH}$.

\section{Proving Correct BSML Implementation of BH in Coq}

From the axioms presented in subsection $\mathrm{V}-\mathrm{A}$ above, we obtain four Coq functions that verify the BSML specifications. These functions and their properties are used when we prove the correctness of a parallel version of $\mathrm{BH}$ with respect to definition 1.

The main theorem is:

Theorem bh_bsml_bh Ist: $\forall($ L A R B:Set $)(k: L \rightarrow A \rightarrow R \rightarrow B)$

(gl: list $A \rightarrow L)$ (opl: $L \rightarrow L \rightarrow L)$ (gr:list $A \rightarrow R$ ) (opr: $R \rightarrow R \rightarrow R$ )

(gl_hom : is_homomorphism A L gl opl)

(gr_hom : is_homomorphism A R gr opr)

(Ist:list A),

list_of_par_list(bh_bsml_comp (gl nil) (scatter lst) (gr nil))) = bh_comp k gl opl gr opr (gl nil) lst (gr nil).

It states the equivalence of bh_comp and the parallel version bh_bsml_comp. The bh_bsml_comp function is quite similar to the direct implementation of BH in BSML given in Fig. 4 This function takes a distributed list (or parallel vector of lists) as input (type 'a list par in BSML and par(list A) in Coq), and also returns a distributed list whereas bh_comp takes as input a list and returns a list. Thus some conversions are needed. scatter takes a list and cuts it into pieces that are distributed. list_of_par_list does the inverse: it takes a parallel vector of lists converts it to a function from natural to lists (using a variant of the proj primitive) and eventually merges the lists into one list.

In order to prove this theorem, two intermediate results are necessary. They state that at a given processor, the Coq formalisation of $\mathrm{vl}$ and $\mathrm{vr}$ in Fig. 4 are respectively equal to applying gl (resp. gr) to the sub-list of elements being on the left (resp. the right) of the local sub-list. The proofs are technical and use several steps where sub-lists are cut and combined. The proofs (available in [27]) are done by considering the processor list as being the list $(11++\mathrm{p}:: n i l)++12$ and by reasoning by induction on $\mathrm{I} 1$ for $\mathrm{vl}$ and on $\mathrm{I} 2$ for $\mathrm{vr}$.

\section{BSP Cost of Parallel BH}

The BSML implementation of $\mathrm{BH}$ applied to parameters $k, g l, \oplus_{l}, g r, \otimes_{r}$ and lst (assumed to be values) has the following complexity: The computation is done in one full super-step followed by some asynchronous local computations. We assume each processor $i$ has a contiguous part of the list $l s t$, that will be denoted by $l s t_{i}$.

The BSP cost of the application of $\mathrm{BH}$ is:

$$
\operatorname{seq}_{1}+\max _{0 \leq i<\text { bsp_p }} \max \left(h_{i}^{+}, h_{i}^{-}\right) \times g+L+s e q_{2}
$$

The first phase computes on each processor, the two "summaries" of the values held by the processor: One to be sent to processors at its left (computed with $g l$ ), and one to be sent to the processors at its right (computed with $g r$ ):

$$
s e q_{1}=\max _{0 \leq i<\mathrm{bsp} \_\mathrm{p}}\left(\overline{g l l s t_{i}}+\overline{g r l s t_{i}}\right)
$$

After that, each processor $i$ sends $l_{i}=\left(g l l s t_{i}\right) \downarrow$ to processors with smaller processor names and $r_{i}=\left(g l l s t_{i}\right) \downarrow$ to processors with greater processor names. So the size of exchanged data is:

$$
\left\{\begin{array}{l}
h_{i}^{+}=i \times\left|l_{i}\right|+(\text { bsp_p }-1-i) \times\left|r_{i}\right| \\
h_{i}^{-}=\sum_{j=0}^{i-1}\left|r_{j}\right|+\sum_{j=i+1}^{\text {bsp_p-1 }}\left|l_{j}\right|
\end{array}\right.
$$

The remaining asynchronous local computation proceeds as follows: First, on each processor the received list of left (resp. right) summaries is reduced with $\oplus_{l}$ (resp. $\left.\otimes_{r}\right)$. Then a local sequential $\mathrm{BH}$ is performed: It is implemented as the computation of the local left and right summaries for each element of the local list yielding to lists $l l_{i}$ and $l r_{i}$. However each of these lists can be computed with traversing only once the local list $l s t_{i}$. The computation ends with apply $k$ to each triple of the list obtained as the combination of the three lists $l l_{i}, l s t_{i}$, and $l r_{i}$. We do not detail the cost in the general case. In cases where $\oplus_{l}, \otimes_{r}$, and $k$ have constant complexity, and $g r$ and $g l$ have constant complexity on singleton lists, we have: seq $_{2} \in \mathcal{O}\left(\right.$ length lst l $_{i}$.

Tower building complexity: With the example of tower building, $\oplus_{l}=\otimes_{r}=\uparrow$ has constant complexity providing that we can do a comparison in constant time. maxAngleL and maxAngle $R$ have the same complexity:

$$
\overline{\text { maxAngleL lst }}=\overline{\text { maxAngleR lst }} \in \mathcal{O}(\text { length lst }) .
$$


So extracted Tower Building has complexity:

$$
\max _{0 \leq i<\text { bsp_p }} \mathcal{O}\left(\text { length lst } t_{i}\right)+g * \mathcal{O}(p)+L
$$

Thus, if the list lst is evenly distributed the complexity is:

$$
\mathcal{O}(\text { length lst } / p)+g * \mathcal{O}(p)+L \text {. }
$$

\section{Programs Extraction and Experiments}

Let us summarise the different steps towards a proved correct parallel implementation of the tower building problem:

(a) First we specify the problem as an instance of mapAround;

(b) using theorem 11, we prove that the problem is an instance of $\mathrm{BH}$; (c) using theorem bh_bsml_bh, we prove that the problem is an instance of the parallel version of $\mathrm{BH}$.

From this latter proof, we can extract an implementation of the tower building problem in BSML. The resulting code is of course similar in structure of the code of the direct implementation of BH in BSML (Fig. (4). The main differences are on the sequential data structures. The lists type are the one defined inductively in Coq, not the optimised ones defined in Ocaml. The BSML primitives in Coq manipulate processor and nat which rely on a Peano encoding of naturals. Thus the extracted code contains: type nat $=|\mathrm{O}| \mathrm{S}$ of nat which is very inefficient for computations.

To test the differences of efficiency between extracted and non-extracted programs, we experimented with two different tower building programs: The direct implementation and the implementation extracted from the derivation in Coq. The experiments were conducted on the "Centre de Calcul Scientifique de la Région Centre (CCSC)" which is a 42 nodes cluster of IBM blades with 2 quad-core Xeon E5450 and $8 \mathrm{~Gb}$ of memory per blade.

We had to use only one core per CPU due to a dramatic loss of performance in MPI communications when multiple cores of the same CPU try to access to the network, and we were able to book up to 18 nodes of the cluster.

In order to avoid the garbage collector of OCaml to be triggered too often, we grew the minor heap size to $1 \mathrm{~Gb}$. We performed a garbage collection after the computation and took its time into account in our benchmark. Indeed, when the data are to big to fit in the minor heap, the recurrent calls to the garbage collector dramatically hinder the overall performance.

Figure 5 shows, for 12 processors the computation time for different sizes of data. We can see that the programs execution time (and the garbage collection time) grows linearly with the amount of data. The extracted version of the program is slower than the direct implementation with a time factor between 1 and 2.5. As said earlier, this come most probably from the difference in data structure encoding.

As shown by figure6 the speedup of both implementation is linear with the number of processors, for a fixed amount of data (5.120.000 elements).

We also performed experiments on the Maximum Prefix Sum example on another cluster, up to 32 processors. Figure 7 also shows a linear speedup (320.000 elements).



Fig. 5

TOWER BUILDING TIMINGS (IN $s$ )

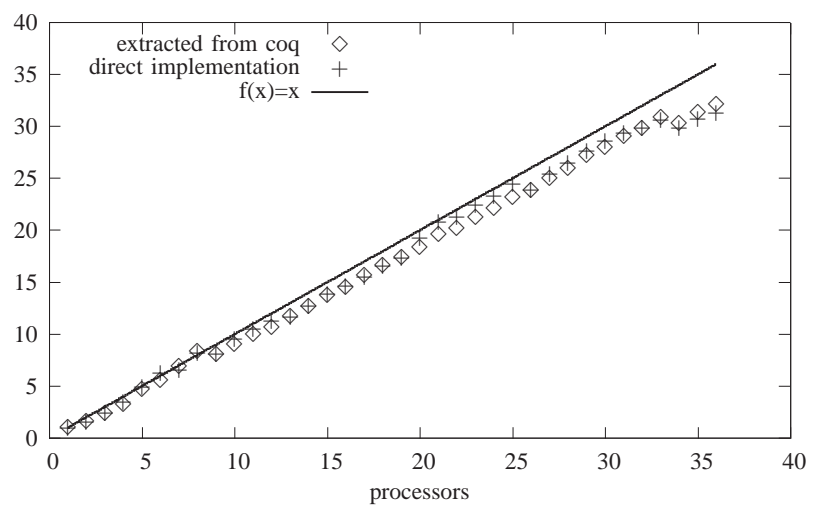

Fig. 6

TOWER BUILDING SPEEDUP

\section{RELATED WORK}

Our framework combines two strength: constructive algorithmic and proved correct bulk synchronous parallel language. In this paper we focus on the semantics of the programming model of Bulk Synchronous Parallel ML, which was first express as an extension of the $\lambda$-calculus [19]. It is possible to implement this semantics as a sequential program, for example by realizing parallel vectors by lists or arrays. But as it is traditional in data-parallel languages, we also provide the semantics of an execution model which describes the parallel implementation of BSML programs as SPMD programs. We even propose a semantics which is even closer to the real implementation: a parallel abstract machine. All these semantics have been proved equivalent [9]. Thus proving the correctness of a BSML program using the semantics of the

Fig. 7

MAXIMUM PREFIX SUM SPEEdUP 
programming model of BSML ensures the parallel execution of this program will also be correct (up to the correctness of the implementation of the parallel abstract machine).

But BSML relies, for communications, on the put operation which is not very easy to use. On the contrary, constructive algorithmic provides various ways to help the programmer of parallel applications to systematically derive efficient parallel algorithms [7], [12]. However there is a gap between the final composition of algorithmic skeletons obtain by derivation and its implementation. Usually in skeletal parallelism, the semantics of the execution model remains informal. Exception are [1], [5]. Several researchers worked on formal semantics for BSP computations, for example [17], [23], [25]. But to our knowledge none of these semantics was used to generate programs as the last step of a systematic development.

On the contrary LOGS [6] is a semantics of BSP programs and was used to generate $\mathrm{C}$ programs [30]. Compared to our approach, there is a gap between the primitives of the semantics and the implementation in C. Our programs are extracted from Coq proof terms. There exists work on bulk synchronous parallel algorithmic skeletons [13], [29]: in these approaches the derivation or optimisation process is guided by the BSP cost model, but the gap between the final composition of skeletons and the implementation is still there.

It is worth noting that the idea of using formal approaches for transforming abstract specifications into concrete implementations was also proposed as abstract parallel machines in [21]. Compared with the study, the contribution of the paper is that we proposed and realized a concrete framework based on the Coq proof assistant and the BSP model.

\section{CONCLUSION}

In this paper, we report our first attempt of combining the theory of constructive algorithmic and proved correct BSML parallel programs for systematic development of certified BSP parallel programs, and demonstrate how it can be useful to develop certified BSP parallel programs. Our newly proposed framework for the certified development of programs includes the new theory for the $\mathrm{BH}$ homomorphism, and an integration of Coq (for specification and development interaction), the $\mathrm{BH}$ homomorphism and BSML programming. All the certification of the transition from specification to algorithms in $\mathrm{BH}$ and to certified BSML parallel programs is done with the Coq proof assistant. We prove, in Coq, theorems validating the transformations of a simple, sequential specification into a more detailed and complex parallel specification. Then, using the program-extraction features of Coq yields a certified parallel program.

\section{REFERENCES}

[1] M. Aldinucci and M. Danelutto. Skeleton-based parallel programming: Functional and parallel semantics in a single shot. Computer Languages, Systems and Structures, 33(3-4):179-192, 2007.

[2] Y. Bertot. Coq in a hurry, 2006. http://hal.inria.fr/inria-00001173.

[3] Y. Bertot and P. Castéran. Interactive Theorem Proving and Program Development. Springer, 2004.

[4] G. E. Blelloch. Scans as primitive operations. IEEE Trans. on Computers, 38(11):1526-1538, 1989
[5] A. Cavarra, E. Riccobene, and A. Zavanella. A formal model for the parallel semantics of p31. In SAC '00: Proceedings of the 2000 ACM symposium on Applied computing, pages 804-812. ACM, 2000.

[6] Y. Chen and J. W. Sanders. Logic of global synchrony. ACM Transaction on Programming Languages and Systems, 26(2):221-262, 2004.

[7] M. Cole. Parallel programming with list homomorphisms. Parallel Processing Letters, 5(2):191-203, 1995.

[8] F. Loulergue et al. The BSML Library version 0.4 . https://traclifo.univ-orleans.fr/BSML 2008.

[9] F. Gava. Approches fonctionnelles de la programmation paralléle et des méta-ordinateurs. Sémantiques, implantations et certification. $\mathrm{PhD}$ thesis, University Paris Val-de-Marne, LACL, 2005.

[10] A. V. Gerbessiotis and L. G. Valiant. Direct bulk-synchronous parallel algorithms. Journal of Parallel and Distributed Computing, 22(2):251267, 1994.

[11] S. Gorlatch. Systematic efficient parallelization of scan and other list homomorphisms. In Euro-Par'96. Parallel Processing, LNCS 1124, pages 401-408. Springer-Verlag, 1996.

[12] S. Gorlatch. Systematic extraction and implementation of divide-andconquer parallelism. In Proc. Conference on Programming Languages: Implementation, Logics and Programs, LNCS 1140, pages 274-288. Springer-Verlag, 1996.

[13] Y. Hayashi and M. Cole. Automated cost analysis of a parallel maximum segment sum program derivation. Parallel Processing Letters, 12(1):95$111,2002$.

[14] Z. Hu, H. Iwasaki, and M. Takeichi. Formal derivation of efficient parallel programs by construction of list homomorphisms. ACM Transactions on Programming Languages and Systems, 19(3):444-461, 1997.

[15] Z. Hu, H. Iwasaki, and M. Takeichi. An accumulative parallel skeleton for all. In 11st European Symposium on Programming (ESOP 2002), LNCS 2305, pages 83-97. Springer, 2002.

[16] Z. Hu, M. Takeichi, and W.-N. Chin. Parallelization in calculational forms. In 25th ACM Symposium on Principles of Programming Languages (POPL'98), pages 316-328. ACM Press, 1998.

[17] H. Jifeng, Q. Miller, and L. Chen. Algebraic laws for BSP programming. In Euro-Par'96. Parallel Processing, LNCS 1123-1124. Springer, 1996.

[18] R. Loogen, Y. Ortega-Mallén, R. Peña, S. Priebe, and F. Rubio. Parallelism Abstractions in Eden. In Patterns and Skeletons for Parallel and Distributed Computing, LNCS 2011. Springer, 2002.

[19] F. Loulergue, G. Hains, and C. Foisy. A Calculus of Functional BSP Programs. Science of Computer Programming, 37(1-3):253-277, 2000.

[20] K. Morita, A. Morihata, K. Matsuzaki, Z. Hu, and M. Takeichi. Automatic inversion generates divide-and-conquer parallel programs. In ACM SIGPLAN 2007 Conference on Programming Language Design and Implementation (PLDI 2007), pages 146-155. ACM Press, 2007.

[21] J. O'Donnell and G. Rünger. Abstract parallel machines. Computers and Artificial Intelligence, 19(2), 2000.

[22] D. Skillicorn. Foundations of Parallel Programming. Cambridge University Press, 1994

[23] D. Skillicorn. Building BSP Progams Using the Refinement Calculus. In Workshop on Formal Methods for Parallel Programming: Theory and Applications, LNCS, pages 790-795. Springer, 1998.

[24] M. Sozeau. Coq 8.2 Reference Manual, chapter Type Classes. INRIA TypiCal, 2008.

[25] A. Stewart, M. Clint, and J. Gabarró. Barrier synchronisation: Axiomatisation and relaxation. Formal Aspects of Computing, 16(1):36-50, 2004.

[26] The Coq Development Team. The Coq Proof Assistant. http://coq.inria.fr

[27] The SDPP Development Team. Systematic Development of Parallel Programs. https://traclifo.univ-orleans.fr/SDPP

[28] L. G. Valiant. A bridging model for parallel computation. CACM, 33(8):103, 1990.

[29] A. Zavanella. Skeletons, BSP and performance portability. Parallel Processing Letters, 11(4):393-405, 2001.

[30] J. Zhou and Y. Chen. Generating C code from LOGS specifications. In 2nd International Colloquium on Theoretical Aspects of Computing (ICTAC'05), LNCS 3407, pages 195-210. Springer, 2005.

\section{APPENDIX}

\section{A Very Short Introduction to COQ}

The Coq proof assistant [26] is based on the calculus on inductive construction. This calculus is a higher-order typed 
$\lambda$-calculus. Theorems are types and their proofs are terms of the calculus. The Coq systems helps the user to build the proof terms and offers a language of tactics to do so.

We illustrate quickly all these notions on a short example :

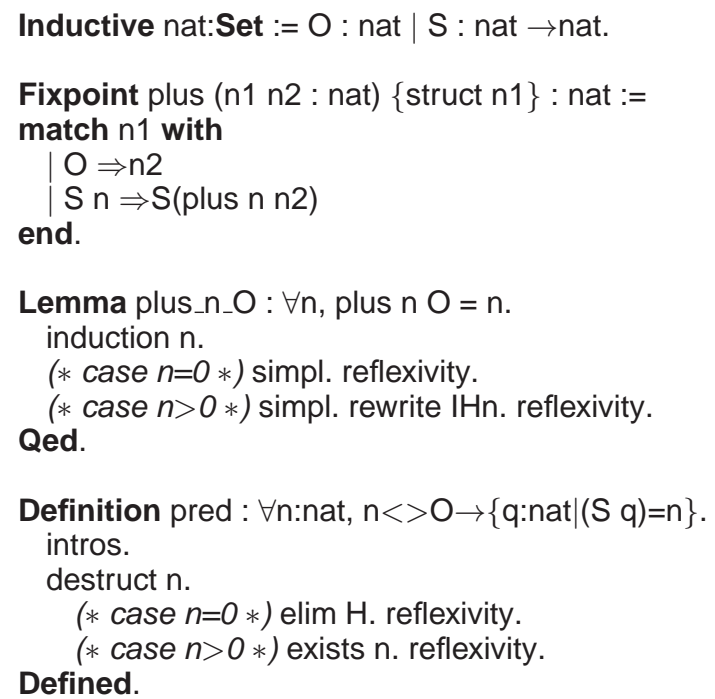

In this example, we first define a new inductive type, the type of natural numbers in the Peano style. nat has type Set which means it belongs the computational realm of the Coq language. We also define the plus recursive function on naturals. In this recursive definition we specify the decreasing argument (here $\mathrm{n} 1$ ) as all functions must be terminating in Coq. In both cases, we gave the type of the new name we wanted to define as well as a term of this type.

We then define a lemma named plus_n_O which states that $\forall n$, plus $\mathrm{nO}=\mathrm{n}$. If we check (using the Check command of Coq) the type of expression, we would obtain Prop which mean that this expression belongs to the logical realm. To define plus_n_O we also should provide a term of this type, that is a proof of this lemma. We could write directly such a term, but it is usually complicated and Coq provides a language of tactics to help the user to build a proof term. If we give to Coq top-level the line beginning with Lemma we would enter the interactive proof mode that would indicate us that we should prove the goal:

$$
\text { forall } \mathrm{n} \text { : nat, plus } \mathrm{n} O=\mathrm{n}
$$

We prove this goal by induction on $\mathrm{n}$ using the tactic induction $\mathrm{n}$. The system indicates now two goals to prove:



The first one is proved using the definition of plus using the tactic simpl which yields the goal $0=0$ and this case is ended by the application of the tactic reflexivity. The second one is the inductive case:

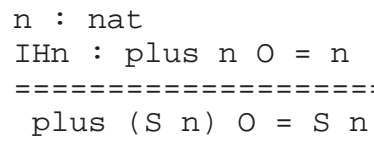

After simplification, we obtain the goal $S($ plus $n O)=S n$. We solve it first by rewriting plus $\mathrm{n} O$ in $n$ using the $\mathrm{IHn}$ hypothesis and then we conclude by reflexivity.

Mixing logical and computational parts is possible in Coq. For example a function of type $A \rightarrow B$ with a precondition $P$ and a postcondition $Q$ corresponds to a constructive proof of type: $\forall x: A,(P x) \rightarrow$ exists $y: B \rightarrow(Q \times y)$. This could be express in Coq using the inductive type sig:

Inductive sig (A:Set) (Q:A $\rightarrow$ Prop) : Set $:=\mid$ exist: $\forall$ $(x: A),(Q x) \rightarrow(\operatorname{sig} A Q)$.

It could also be written, using syntactic sugar, as $\{\mathrm{x}: \mathrm{A} \mid(\mathrm{P} x)\}$.

This feature is used in definition of the function pred. The specification of this function is: $\forall \mathrm{n}: n a t, \mathrm{n}<>\mathrm{O} \rightarrow\{\mathrm{q}: \mathrm{nat} \mid(\mathrm{S} \mathrm{q})=\mathrm{n}\} \quad$ and we build it using tactics. We reason by case on $\mathrm{n}$ (tactic destruct). The first case is easily solved because we have the hypothesis $0<>0$, the second one is trivial.

The command Extraction pred would extract the computational part of the definition of pred. We could obtain a certified implementation of the predecessor function:

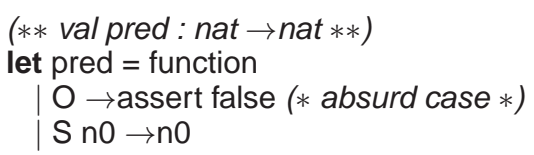

[2] is a quick yet longer introduction to Coq. 